\title{
XLIX. Description of the photographometer, an instrument for measuring the intensity of the chemical action of the rays of light on all the photographic preparations, and for comparing with each other the sensitiveness of these different preparations
}

\section{A. Claudet}

To cite this article: A. Claudet (1848) XLIX. Description of the photographometer, an instrument for measuring the intensity of the chemical action of the rays of light on all the photographic preparations, and for comparing with each other the sensitiveness of these different preparations, Philosophical Magazine Series 3, 33:223, 329-335, DOI: 10.1080/14786444808646114

To link to this article: http://dx.doi.org/10.1080/14786444808646114

Published online: 30 Apr 2009.

Submit your article to this journal $\longleftarrow$

Џll Article views: 3 
T H E

LONDON, EDINBURGH AND DUBLIN

PHILOSOPHICAL MAGAZINE

A N D

JOURNAL OF SCIENCE.

[THIRD SERIES.]

NOVEMBER 184.8.

XLIX. Description of the Photographometer, an instrument for measuring the Intensity of the Chemical Action of the Rays of Light on all the Photographic preparations, and for comparing with each other the sensitiveness of these different preparations. Invented by A. Claudet, London, September $1848 *$.

[With a Plate.]

WTE are not yet in possession of an instrument capable of measuring the variations in intensity of the sun's light with the same precision that we appreciate those of its heat. In photographic operations the want of a photometer has long been felt; and it was soon perceived that it was not sufficient to measure the light, properly so called, but certain accompanying rays, which exert on different bodies a chemical action without producing on the retina the sensation by means of which objects appear to us visible. All photographers know by experience that the chemical action is not exactly in proportion to the intensity of the visible light.

Many ingenious contrivances have been resorted to for indicating the intensity of the chemical or actinic rays existing at a given moment; but as the object was to study more particularly the variations of the direct solar rays, it was found necessary to have recourse to complicated instruments furnished with heliostats and clock-work movements.

In $1839 \mathrm{Mr}$. 'T. B. Jordan of Falmouth invented an instrument which he called a heliograph. This instrument was imperfect. It was modified and improved by $\mathrm{Mr}$. R. Hunt, who has devoted much time to photographic processes and researches on the properties of the different rays of light.

The apparatus of Mr. Hunt, which he called an actinograph, consists of a cylinder placed parallel to the axis of the

* This paper has been communicated to the Académie des Sciences, October 9.

Phil. Mag. S. 3. Vol. 33. No. 223. Nov. 1848. 
ecliptic, having a triangular opening and turning upon its axis, following the sun's path, clock-work movement being used for the purpose.

The photogenic paper is rolled upon another cylinder placed in the interior of the first, and turning in a contrary direction. The sun's rays, passing through the triangular opening, fall successively upon all that portion of the paper exposed by the rotation of the exterior cylinder.

The photogenic effect produced upon the paper is in direct proportion to the intensity of the light present during the motion of the triangular opening, and also in direct proportion to the length of the lines parallel to the base of the triangle at every part of its opening; so that as the intensity of the light increases or diminishes, the commencement of the photogenic effect is indicated at a point more or less distant from the base. At the end of the day, on removing the paper we find a long figure, the height of the ordinates of which is the measure of the intensity of the chemical action of the light.

Mr. Hunt described his actinograph at the meeting of the British Association at Cambridge in June 1845.

Sir John Herschel in 1840 constructed an instrument for the same purpose. He places photogenic paper under an immoveable disc, having a narrow opening in the direction of its radius for the passage of the sun's light reflected by a heliostat: this light falls on the paper fixed upon another disc, turning on its axis by clock-work.

At the end of the day every part of the photogenic paper has passed this opening, and the effect produced shows theintensity of the light during the various hours from sunrise to sunset.

All these several ingenious contrivances had no other object than that of measuring the photogenic intensity of the direct rays of the sun during the different hours of the day. Besides, they were applicable only to photography on paper; and the results were uncertain, because the paper does not long preserve the same degree of sensitiveness: then the heliostats and clock-work movements rendered the apparatus complicated, expensive and difficult to use.

It may then be asserted, that up to the present time there does not exist an instrument for indicating to the photographer the intensity of the chemical rays, and at the same time the sensitiveness of his preparation.

I have endeavoured to supply this want, and offer the result of my labours in publishing an instrument which, I believe, fulfils all the conditions necessary to render it useful, as it measures at the same time the intensity of the chemical rays and the sensitiveness of the preparation. I have called it a 
photographometer, because it indicates the combined results of the photographic operation.

My apparatus is very simple, and serves equally for processes on paper or on metallic plates. It indicates the intensity of the chemical rays at all moments of the day during atmospheric variations, and at the instant we may wish to operate. It serves also to compare the degree of sensitiveness of the different photographic preparations.

For an instrument of this kind (see Plate II. fig. 1), it is important in the first place to have a motion always uniform, without complicated or expensive mechanism. This I have obtained by a means founded upon the principle of the fall of bodies sliding down an inclined plane. The sensitive surface is exposed to the light by the rapid and uniform passage of a metal plate $A B$, fig. 2, having openings of different lengths which follow a geometric progression. It is evident that the exposure to light will be the same for each experiment, because the plate furnished with the proportional openings falls always with the same rapidity, the height of the fall being constant, and the angle of the inclined plane the same. Each opening of this moveable plate allows the light to pass during the same space of time, and the effect upon the sensitive surface indicates exactly the intensity of the chemical rays. The rapidity of the fall may be augmented or diminished by altering the inclination of the plane by means of a graduated arc $\mathrm{CD}$, fig. 1, furnished with a screw $\mathrm{E}$, by which it may be fixed at any angle. The same result may be obtained by modifying the height of the fall or the weight of the moveable plate. The photogenic surface, whether it be the Daguerreotype plate, the Talbotype paper, or any other preparation sensitive to light, is placed near the bottom of the inclined plane F, figs. $1 \& 2$. It is covered by a thin plate of metal pierced with circular holes, which correspond to the openings of the moveable plate at the moment of the passage of the latter, during which the sensitive surface receives the light wherever the circular holes leave it exposed.

The part of the apparatus which contains the sensitive surface is an independent frame, and it slides from a dark box into an opening on the side of the inclined plane. The fig. 3 represents the frame and the dark box, and by its inspection the manner will be understood in which the sensitive surface is placed in it before the experiment and carried to the mercury-box after the operation.

A covering of black cloth impermeable to light is attached to the sides of the moveable plate enveloping the whole inclined plane, rolling freely over two rollers $R, R^{\prime}$ placed, the 
one at the upper and the other at the lower part of the inclined plane. This cloth prevents the light striking the sensitive surface before and after the passage of the moveable plate.

The openings of the moveable plate are parallel to each other, and in the direction of the inclined plane. They are seven in number. The first is 1 millimetre; the second, 2 millim.; the third, 4 millim.; the fourth, 8 millim.; the fifth, 16 millim.; the sixth, 32 millim.; and the seventh, 64 millim. Each opening is then the half of the following, and double of the preceding one. After the operation we have seven (or less, according to the intensity of light) separate images, the different intensities of which represent the action of the light during intervals of time in the geometric progression of $:: 1$ : $2: 4: 8: 16: 32: 64$. These numbers are quite sufficient for general observation; we can multiply the effects by allowing the plate to fall twice, thrice, or a greater number of times. I adopted this progression because I found an arithmetic progression did not give an appreciable difference in the intensity of the photogenic effect, a doubling of the action being required to produce a marked difference of effect. If we wish to compare the photogenic intensity of the sun's rays with those of the moon, we allow the moveable plate to fall a number of times sufficient for the moon's rays to produce an action, which may be rendered apparent by the mercury. A few trials suffice to obtain an effect from which may be calculated the proportion of the intensities.

The moveable plate containing the parallel proportionate openings is furnished with a flap which opens and shuts freely, and by which the openings may be protected from light when the plate is to be raised again in order to fall a second time.

A simple apparatus thus constructed will be a photographometer certain for every preparation. It will be an index and an infallible guide for the operator. It will indicate the intensity of the photogenic light, and also the sensitiveness of the preparation. But it becomes still more useful when constructed double; that is to say, when the moveable plate is furnished with two equal series of proportional openings placed in a line perpendicular to the direction of the inclined plane, and the fixed plate pierced with two series of holes corresponding to the openings in the moveable plate.

By placing beneath each series of holes a different sensitive surface, each of these surfaces will, during the fall of the moveable plate, receive the same proportion of the same light, and thus their different degrees of sensitiveness may be compared. In this manner we learn the comparative sensitiveness of different preparations of the iodide, of the bromo-iodide and 
chloro-iodide of silver, and of the various photogenic papers; for it is indispensable, in making an exact comparison, to operate with the same light and during strictly the same space of time, as it is known that the light varies from one minute to another.

When we only wish to make experiments of comparison with different preparations, a much more simple and portable apparatus will serve as well. In this the plate furnished with parallel openings is immoveable, and fixed in the centre of a frame having grooves underneath to keep it in contact with another frame, in which we place the two sensitive surfaces we wish to compare. Two plates, having round holes for the passage of the light, cover the sensitive surfaces, which are held by a shutter fastened at the back by a button. This part of the apparatus resembles a Daguerreotype " passe partout," with this difference, that in the place of the glass we have a metallic plate furnished with holes.

When the apparatus has been charged in a room shaded from the daylight, it is carried out, and the frame containing the sensitive surfaces is made to slide along by the hand, while the frame containing the proportional openings is exposed to the daylight or to the sun. With a little practice, a movement may be obtained regular enough to measure by this simple apparatus, with sufficient exactness, the intensity of the light.

By the double apparatus, whether it has the uniform motion given by the inclined plane, or that less regular given by the hand, we shall decide a very interesting question; which is, to know if each variation in the quantity and quality of light affects equally the different photogenic preparations. We shall find, perhaps, that certain lights require different preparations or proportions to obtain the maximum of sensitiveness.

In place of a single series of round holes upon each plate, we can have two, three, four or five, which enable us to make several experiments upon the same surface. On the paper, for instance, we can prepare a zone for each series of holes with a different substance; and on the Daguerreotype plate we may vary the coatings of iodine, bromine or chlorine, so that the same surface will contain two, three, four, or five experiments of comparison, serving to indicate the best method of preparation.

The plate, fig. 4, shows the result of an experiment of this kind, and is the exact representation of the plate itself after it has received the mercurial vapour. The plate had been exposed to the vapour of iodine in such a manner that one zone had attained the first coating of yellow colour, a second zone had reached the red, a third the blue-green, and the fourth, after having passed through all these tints, had obtained the 
second yellow coating. The number of white circular spots on each vertical zone indicates the degree of sensitiveness of these various coatings; the less sensitive being the first coating of yellow, and the most sensitive the second coating of the same colour, which I call the double coating of iodine.

Therefore, we have an instrument by which we can decide, in an incontestable manner, upon the merits of different accelerating liquids used in the Daguerreotype, resolve a great number of problems, and make experiments, from which will infallibly result the simplification and improvement of the art of photography, which up to the present time has suffered too much from empiricism and charlatanism.

Appended to this description are a drawing of the apparatus, and the plans necessary to explain its action and construction. (See Plate II.)

I am still engaged in making experiments with this apparatus; as soon as they are completed I shall hasten to communicate the result of my labours. I content myself for the present with announcing a very extraordinary fact which my apparatus has furnished me with.

I do not give it as the result of a calculation mathematically correct; but I cannot be far from the truth in stating, that the pure light of the sun modifies the bromo-iodized silver plate, communicating to it an affinity for mercurial vapour which produces the white image in the Daguerreotype, in a space of time which cannot be much more than the thousandth part of a second. I made the experiment in the following manner :-I let the light of the sun fall upon the plate through an opening of a millimetre, whilst this opening passed over a space of 250 millimetres in one quarter of a second, as near as I could judge; this light could not therefore have acted on the plate during much more than the $\frac{1}{100} \overline{0}$ dth part of a second, and nevertheless this inconceivably short space of time sufficed to produce a decided effect.

It is not necessary that I should indicate all the applications of this photographometer, and the experiments which may be made with it. Without doubt many will be discovered which have not occurred to me; but I will enumerate a few which appear to me important enough to merit the attention of philosophers:- What is the effect of the compound ligh and that of the different separated rays of the solar spectrum? How much photogenic light is lost by reflexion from parallel mirrors, prisms and other substances, and by refraction through lenses? The proportion of photogenic rays in the lights obtained from various sources, including that produced by electricity? If the photogenic light varies with the height of the atmosphere and with the changes of temperature? If 
On the Extension of the Theorem of Leibnitz to Integration. 335

it is affected by the electrical state of the atmosphere? In fine, what is the proportion of the photogenic rays at each hour of the day, and at different points in space at a given moment 3

L. On the Extension of the Theorem of Leibnitz to Integration. By J. R. Young, Professor of Mathematics, Belfast*.

THE following method of showing the applicability of the 1 theorem of Leibnitz to successive integration, though new to me, may possibly be found in some works on the integral calculus which I have not seen. I venture however to give it here, chiefly because it suggests a consideration, in reference to the "Calculus of Operations," which is perhaps deserving of notice.

If we put $d u_{1}$ for $u d x, d u_{2}$ for $u_{1} d x, d u_{3}$ for $u_{2} d x$, and so on, and apply " integration by parts" to the differential vudx, we shall have

$$
\begin{aligned}
\int v u d x & =v u_{1}-\int \frac{d v}{d x} u_{1} d x \\
& =v u_{1}-\frac{d v}{d x} u_{2}+\int \frac{d^{2} v}{d x^{2}} u_{2} d x \\
& =v u_{1}-\frac{d v}{d x} u_{2}+\frac{d^{2} v}{d x^{2}} u_{3}-\int \frac{d^{3} v}{d x^{3}} u_{3} d x \\
& =v u_{1}-\frac{d v}{d x} u_{2}+\frac{d^{2} v}{d x^{2}} u_{3}-\frac{d^{3} v}{d x^{3}} u_{4}+\ldots \ldots \frac{d^{m-1} v}{d x^{m-1}} u_{m} \\
& \pm \int \frac{d^{m} v}{d x^{m}} u_{m} d x
\end{aligned}
$$

the sign of this last quantity being opposite to that of the term immediately preceding it, and which term, here regarded as the $m$ th term, may be any whatever in the series. Thus far there is nothing new.

Integrating again, and proceeding on the same principles, omitting however the supplementary integral, which, as above, should terminate each of the following rows, we shall have

$$
\begin{array}{r}
\int{ }^{2} v u d x^{2}=v u_{2}-\frac{d v}{d x} u_{3}+\frac{d^{2} v}{d x^{2}} u_{4}-\frac{d^{3} v}{d x^{3}} u_{5}+\ldots . \\
-\frac{d v}{d x} u_{3}+\frac{d^{2} v}{d x^{2}} u_{4}-\frac{d^{3} v}{d x^{3}} u_{5}+\ldots \ldots \\
+\frac{d^{2} v}{d x^{2}} u_{4}-\frac{d^{3} v}{d x^{3}} u_{5}+\ldots \ldots \\
-\frac{d^{3} v}{d x^{3}} u_{5}+\ldots \ldots \\
\& c . \quad \text { \&c. }
\end{array}
$$

* Communicated by the Author. 